\title{
Clinical diagnosis and treatment of breast cancer with brain metastases and establishment of a prognostic model: a 10-year, single-center, real-world study of 559 cases
}

\author{
Limin Niu ${ }^{1}$, Huimin $\mathrm{Lv}^{1}$, Mengwei Zhang ${ }^{1}$, Huiai Zeng ${ }^{1}$, Lifeng Wang ${ }^{2}$, Shude Cui ${ }^{1}$, Zhenzhen Liu ${ }^{1}$, \\ Min Yan $^{1}$ \\ ${ }^{1}$ Department of Breast Disease, Henan Breast Cancer Center, The Affiliated Cancer Hospital of Zhengzhou University \& Henan Cancer Hospital, \\ Zhengzhou, China; ${ }^{2}$ The Affiliated Cancer Hospital of Zhengzhou University \& Henan Cancer Hospital, Zhengzhou, China \\ Contributions: (I) Conception and design: L Niu, S Cui, Z Liu, M Yan; (II) Administrative support: S Cui, Z Liu, M Yan; (III) Provision of study \\ materials or patients: L Niu, H Lv, M Zhang, H Zeng, M Yan; (IV) Collection and assembly of data: L Niu, L Wang; (V) Data analysis and \\ interpretation: L Niu, H Lv, M Yan; (VI) Manuscript writing: All authors; (VII) Final approval of manuscript: All authors. \\ Correspondence to: Min Yan. Department of Breast Disease, Henan Breast Cancer Center, The Affiliated Cancer Hospital of Zhengzhou University \& \\ Henan Cancer Hospital, 127 Dongming Road, Zhengzhou 450008, China. Email: ym200678@126.com.
}

Background: This study involved a retrospective analysis of 559 metastatic breast cancer (MBC) patients with brain metastasis (BM). We aimed to establish the effectiveness of different preferred treatment methods and factors affecting overall survival following BM diagnosis (BMOS) and explore the feasibility of systemic treatment for MBC patients with BM.

Methods: Univariate and multivariate analyses were used to assess the efficacy of different preferred treatments and other factors associated with BMOS, and a nomogram was then established based on the results of the univariate analysis.

Results: Patients that initially received systemic drug therapy exhibited a clinical benefit rate (CBR) of $43.9 \%$ and an intracranial disease control rate (DCR) of $80.6 \%$. The median time between BM diagnosis and the requirement for local intracranial treatment due to worsening disease status was 10.0 months for these patients (95\% CI: 7.811-12.189 months). The median follow-up was 28.0 months, and the median BMOS was 16.0 months. Following BM diagnosis, the systemic drug treatment group had a better outcome than the local brain treatment group, with a respective median BMOS of 22.0 and 16.0 months $\left(\chi^{2}=7.743, \mathrm{P}=0.005\right)$. At the time of BM diagnosis, the median BMOS for patients without neurological symptoms diagnosed by regular screen was significantly longer than that of patients with neurological symptoms (18.0 vs. 13.0 months, respectively; $\chi^{2}=11.371, \mathrm{P}=0.001$ ). Based on these analyses, a nomogram was constructed that incorporated disease-free survival (DFS), Karnofsky performance status (KPS), molecular subtype, number of extracranial metastases, BM location, number of BMs, neurological symptoms, and the preferred treatment approach, with a prediction probability (c-index) value of 0.76 .

Conclusions: Systemic drug treatment has a beneficial effect on brain lesions, and effective treatment delays the need for local intracranial treatment. Cranial magnetic resonance imaging (MRI) screening can detect asymptomatic BM in MBC patients (particularly those with HER2-positive or triple-negative disease), offering these patients an opportunity to undergo systemic drug therapy, thereby prolonging their survival. To our knowledge, this is a well-fitted nomogram including current treatment and medical examination strategies to predict BMOS probability that offers value as an adjunct for the prognostic evaluation of MBCBM patients.

Keywords: Breast cancer; brain metastasis (BM); drug therapy; prognosis; nomogram

Submitted Jun 25, 2021. Accepted for publication Aug 16, 2021.

doi: $10.21037 /$ atm-21-3734

View this article at: https://dx.doi.org/10.21037/atm-21-3734 


\section{Introduction}

Rates of brain metastasis $(\mathrm{BM})$ in breast cancer patients are second only to those in lung cancer patients, with breast cancer accounting for $10-15 \%$ of all BM (1) and with up to $36 \%$ of breast cancer patients found to harbor BM upon autopsy (2). BM incidence varies among different breast cancer molecular subtypes (3); patients with human epidermal growth factor receptor 2 (HER2)-positive and triple-negative breast cancer (TNBC) exhibit higher $\mathrm{BM}$ incidence rates relative to those with other disease subtypes $(4,5)$.

$\mathrm{BM}$ in breast cancer patients is generally associated with a significant reduction in quality of life and a worse prognosis $(6,7)$, with a 1 -year survival rate of just $20 \%(8)$. Regular BM screening is generally believed to have no impact on patient prognosis (9), and ASCO(The Association for Clinical Oncology)guidelines do not recommend routine screening for $\mathrm{BM}$ in those with metastatic breast cancer (MBC) (10). However, early BM diagnosis and symptomatic treatment have been linked to significant improvements in patient prognosis $(7,11-13)$.

As breast cancer treatments continue to progress and become more effective, the average duration of patient survival will improve, and this will almost certainly coincide with an increase in BM incidence $(14,15)$. Even so, no systemic treatment plan for breast cancer-associated BM has yet been approved (10). Currently, major guidelines recommend localized treatment for MBC-BM patients, with systemic treatment only as a supplementary approach (10,16-18). Only patients with certain types of MBC, such as HER2-positive breast cancer with BM, are advised to undergo systemic drug-based therapy.

Both BM and local treatments for BM can cause neurological damage and adversely impact patients' quality of life, and additional brain radiotherapy following enough brain radiotherapy is often difficult to tolerate because of too many side effects. Therefore, it is important to explore the role of systemic drug treatment for MBC$\mathrm{BM}$ patients to highlight novel or more reliable diagnostic and therapeutic strategies for affected individuals. To that end, the present study was designed as a real-world clinical analysis of $559 \mathrm{MBC}-\mathrm{BM}$ patients admitted to Henan Tumor Hospital between January 1, 2010, and December 31, 2019. By comparing the effectiveness of patients who received initially local or systemic treatment and the factors affecting overall survival following BM diagnosis (BMOS), we seek to provide a reference for the diagnosis and treatment of MBC-BM.

The Breast-Graded Prognostic Assessment (B-GPA) $(19,20)$ and the Modified B-GPA (mB-GPA) $(21,22)$ have been proposed as useful tools for stratifying survival in the MBC-BM population. Prognostic factors were assessed by univariate and multivariate Cox proportional hazards regression and analyses. To date, the main prognostic factors $(21,23)$ identified by these methods are the Karnofsky performance score (KPS), age at time of BM diagnosis, BC subtype, number of BMs, and the presence and status of extracranial disease. Prognostic scores for MBC-BM have been progressively upgraded over the last two decades, prompting the clinical need for an increasingly accurate prediction of patient outcomes to adapt treatment strategies accordingly.Recent advances in effective systemic therapies (e.g., anti-HER2 targeted therapy) and radiation therapies have substantially modified and improved BMOS, at least for some subgroups of patients $(21,24)$. Consequently, continuous reassessment and improvement of prognostic tools are urgently needed.

We present the following article in accordance with the STROBE reporting checklist (available at https://dx.doi. org/10.21037/atm-21-3734).

\section{Methods}

\section{Patients}

Patients eligible for inclusion in this study were those meeting the following criteria: (I) patients with $\mathrm{MBC}$ that had been pathologically diagnosed in The Affiliated Cancer Hospital of Zhengzhou University \& Henan Cancer Hospital from January 1, 2010, to December 31, 2019, including male breast cancer patients and individuals with bilateral breast cancer; and (II) patients with BM, including brain parenchymal metastases and meningeal metastases, that had been confirmed by imaging examinations. Patients were excluded from this study if they had any history of other malignancies within the past three years other than cured cervical carcinoma in situ, skin basal cell carcinoma, or squamous cell carcinoma.

Prospective information collected from these patients included demographic information, clinical and pathological characteristics, and the treatment approaches used. Patient outcomes were established by telephonebased follow-up. All procedures performed in this study involving human participants were in accordance with the Declaration of Helsinki (as revised in 2013). This study 
Table 1 Clinical characteristics of 559 patients

\begin{tabular}{|c|c|c|}
\hline Characteristics & Patient (n) & Percentage (\%) \\
\hline \multicolumn{3}{|l|}{ Sex } \\
\hline Female & 557 & 99.6 \\
\hline Male & 2 & 0.4 \\
\hline \multicolumn{3}{|l|}{ Age (years) } \\
\hline$<50$ & 291 & 52.1 \\
\hline$\geq 50$ & 268 & 47.9 \\
\hline \multicolumn{3}{|l|}{ KPS } \\
\hline$\geq 70$ & 340 & 60.8 \\
\hline$<70$ & 219 & 39.2 \\
\hline \multicolumn{3}{|l|}{ Stage of initial diagnosis } \\
\hline 1 & 28 & 5.0 \\
\hline II & 195 & 34.9 \\
\hline III & 208 & 37.2 \\
\hline IV & 98 & 17.5 \\
\hline Unknown & 30 & 5.4 \\
\hline \multicolumn{3}{|l|}{ DFS (months) } \\
\hline$<24$ & 306 & 54.7 \\
\hline$\geq 24$ & 253 & 45.3 \\
\hline \multicolumn{3}{|l|}{ Molecular type } \\
\hline HR+/HER2- & 160 & 28.6 \\
\hline HER2+ & 296 & 53.0 \\
\hline TNBC & 92 & 16.4 \\
\hline Unknown & 11 & 2.0 \\
\hline \multicolumn{3}{|c|}{ Number of extracranial metastatic organs } \\
\hline 0 & 24 & 4.3 \\
\hline $1-2$ & 220 & 39.4 \\
\hline$\geq 3$ & 315 & 56.3 \\
\hline \multicolumn{3}{|l|}{ Site of extracranial metastasis } \\
\hline Soft tissues or lymph nodes & 384 & 68.7 \\
\hline Bones & 312 & 55.8 \\
\hline Livers & 267 & 47.8 \\
\hline Lungs & 309 & 55.3 \\
\hline Others & 201 & 36.0 \\
\hline
\end{tabular}

Table 1 (continued)
Table 1 (continued)

\begin{tabular}{|c|c|c|}
\hline Characteristics & Patient (n) & Percentage (\%) \\
\hline \multicolumn{3}{|l|}{ BM location } \\
\hline Parenchyma & 537 & 96.1 \\
\hline Dura Mater & 35 & 6.3 \\
\hline Pia Mater & 33 & 5.9 \\
\hline \multicolumn{3}{|l|}{ Size of the largest BM (cm) } \\
\hline 0 (meningeal metastasis only) & 22 & 3.9 \\
\hline$<1$ & 222 & 39.7 \\
\hline $1-3$ & 199 & 35.6 \\
\hline$>3$ & 37 & 6.6 \\
\hline Unknown & 79 & 14.1 \\
\hline \multicolumn{3}{|l|}{ Number of BMs } \\
\hline 0 (meningeal metastasis only) & 22 & 3.9 \\
\hline 1 & 166 & 29.7 \\
\hline 2 & 51 & 9.1 \\
\hline 3 & 22 & 3.9 \\
\hline$>3$ & 235 & 42.0 \\
\hline Unknown & 63 & 11.3 \\
\hline \multicolumn{3}{|c|}{ With or without neurological symptoms onset BM } \\
\hline No & 341 & 61.0 \\
\hline Yes & 218 & 39.0 \\
\hline \multicolumn{3}{|l|}{ Preferred brain local treatment } \\
\hline Yes & 330 & 59.0 \\
\hline No & 148 & 26.5 \\
\hline Untreated & 64 & 11.4 \\
\hline Unknown & 17 & 3.0 \\
\hline
\end{tabular}

KPS, Karnofsky performance status; DFS, disease-free survival; HR, hormone receptor; HER2, human epidermal growth factor receptor 2; TNBC, triple-negative breast cancer; BM, brain metastases.

was approved by the medical ethics committee of The Affiliated Cancer Hospital of Zhengzhou University \& Henan Cancer Hospital (No. 2018160). Informed consent from patients was waived due to the retrospective nature of this study. In total, of whom,559 patients were included in the present study (Table 1). Of whom 160 had hormone 


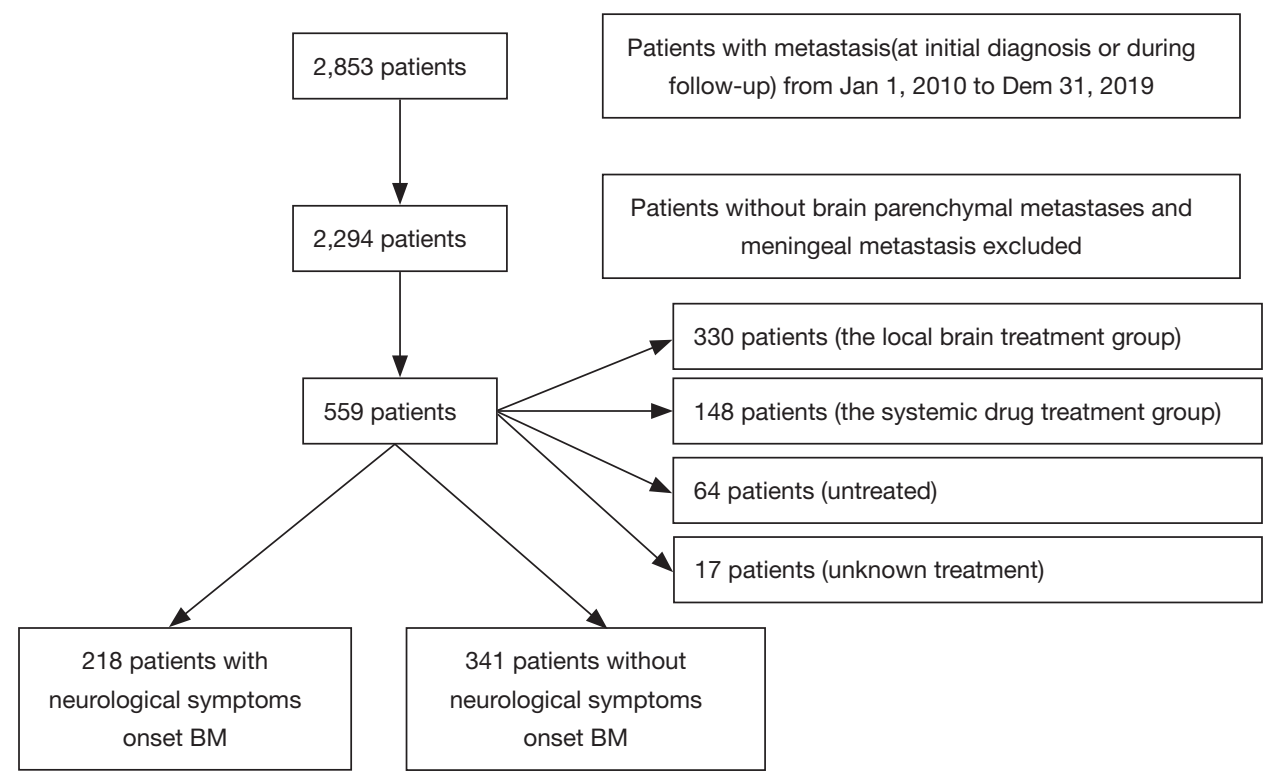

Figure 1 A flow diagram: patient selection of 559 cases.

receptor (HR)+/HER2 - disease, 296 had HER2+ disease, and 92 were diagnosed with TNBC. Of these patients, 537 exhibited brain parenchymal metastases while 33 exhibited leptomeningeal metastasis. Additionally, 239 patients exhibited $\leq 2$ BMs in number, while 257 exhibited $\geq 3$ BMs. Furthermore, on initial diagnosis, 218 cases exhibited neurological symptoms while 341 cases were asymptomatic. A total of 148 cases were initially treated with systemic drug therapy, and 330 cases underwent local treatment (Figure 1).

\section{Study definitions}

Disease-free survival (DFS) was defined as the time from surgery to disease recurrence or all-cause death. BMOS was defined as the duration of overall survival (OS) following BM diagnosis, measured until death or the most recent follow-up. The BM-free interval was the time from initial breast cancer diagnosis to BM diagnosis. OS after metastasis was the time from MBC diagnosis to death or the most recent follow-up.

As per the Response Evaluation Criteria in Solid Tumors version 1.1, treatment efficacy was classified as complete response (CR), partial response (PR), stable disease (SD), or progressive disease (PD). The objective response rate (ORR) was defined as the ratio of $\mathrm{CR}+\mathrm{PR}$ cases to total cases, the clinical benefit rate (CBR) was defined as the ratio of $\mathrm{CR}+\mathrm{PR}+\mathrm{SD}$ cases $(\geq 24$ weeks) to total cases, and the disease control rate (DCR) was defined as the ratio of $\mathrm{CR}+\mathrm{PR}+\mathrm{SD}$ cases to total cases.

\section{BM diagnosis and follow-up}

Cranial-enhanced magnetic resonance imaging (MRI) is a more sensitive measure of detecting small lesions, edema, and meningeal metastases than enhanced computed tomography (CT). Cranial plain + enhanced MRI scans were routinely used, with cranial plain + enhanced CT scan analyses recommended for those patients with MRI contraindications.

Brain/spinal cord MRI screening was performed for patients with neurological symptoms to diagnose the presence of brain parenchymal/meningeal/spinal cord metastases. Typical symptoms included unexplained headache, vomiting, sensory or motor peripheral/central nervous system symptoms, and abnormal defecation and urination.

Patients who exhibited initial recurrence and metastasis or extracranial lesion progression during treatment were routinely administered a cranial plain + enhanced MRI scan. Those MBC patients who exhibited stable extracranial lesions during treatment were re-examined via a cranial plain + enhanced MRI scan every 6-12 months, at the same time that can minimize selection bias. When calculating BMOS, patients with brain parenchymal metastasis combined with leptomeningeal metastasis were counted as 
leptomeningeal metastasis patients.

The most recent follow-up data were obtained on June 30, 2020.

\section{MBC-BM treatment}

MBC-BM treatment was performed as per major guidelines and recommendations, with local treatment as preferred treatment and systemic treatment being reserved as a supplementary approach. For individuals with symptomatic BM and patients diagnosed with spinal cord or leptomeningeal metastases, initial treatment included local treatment (surgery or radiotherapy) combined with systemic drug therapy. For those patients not requiring urgent local brain treatment, systemic drug therapy was the preferred choice, and the efficacy of such treatment on extracranial and intracranial lesions was regularly evaluated. Local treatment was performed if intracranial lesion progression was detected.

\section{Statistical analyses}

SPSS 23.0 (www.ibm.com/legal/copytrade.shtml) and R v4.0.3 (http://www.r-project.org; The R Foundation for Statistical Computing, Vienna, Austria) were used in the present study.

The Kaplan-Meier approach was used to assess the survival outcomes of all patients, and the log-rank test was used to analyze differences in prognosis among groups. Factors yielding a $\mathrm{P}$ value $<0.05$ in the initial univariate logistic regression analyses were incorporated into a subsequent multivariate Cox proportional hazard stepwise regression analysis.

A nomogram model was established based on the data of 472 patients whose all factors were known. The nomogram was constructed to identify the prognostic factors affecting $\mathrm{BMOS}$, which were defined as the factors yielding a $\mathrm{P}$ value $<0.05$ in the univariate analyses.

The 472 patients were randomized into a training set ( $n=377$, for nomogram construction) or a validation set $(n=95$, for nomogram validation) at a ratio of $4: 1$. The training group samples were used to establish a logistic regression model to determine the independent risk factors. A fixed seed [226] was set to reproduce the modeling results. When constructing the logistic regression model, the training set was used to assess the eight factors identified as independent variables, with survival status as the dependent variables. After training, the model was used to predict the survival status of patients in the validation cohort, and a confusion matrix was constructed to present the results of these predictive analyses. This matrix revealed a relatively high accuracy rate of $78.9 \%(46+29 / 46+17+3+29)$.

In the nomogram, each variable was assigned a point value ranging from 0 to 100 . A vertical line for each variable was drawn to the top axis of the nomogram, and the points along the 'Points' axis were used to assign a score to this variable. Point values for each variable were summed to determine the total points number. A vertical line was then drawn from the 'Total Points' axis down to the 'Risk of BMOS' axis to predict the BMOS for a given patient. Area under the curve (AUC) values were used to assess the prediction probability of the nomogram. Internal validation of this model was performed using receiver operating characteristic (ROC) curves. Survival rates (0.5-, 1-, and 1.5 -year) were constructed based on the predictive models for the identified prognostic factors. A P value $<0.05$ served as the significance threshold for this study.

\section{Results}

\section{Patient characteristics}

In total, 2,853 MBC patients were admitted to The Affiliated Cancer Hospital of Zhengzhou University \& Henan Cancer Hospital from January 1, 2010, to December 31, 2019, of whom 559 (19.6\%) were diagnosed with BM. Of these cases, 143 (25.6\%) were diagnosed with BM upon initial hospital presentation, whereas 416 (74.4\%) were diagnosed upon follow-up. Of these 559 patients with BM, $46(8.2 \%)$ exhibited brain parenchymal metastases with meningeal metastases, while 22 (3.9\%) exhibited leptomeningeal metastases only, and 17 (3.0\%) presented with spinal cord metastases that had developed after brain parenchymal/meningeal metastasis.

The median patient age at BM diagnosis was 49 years (range: $24-75$ years), and 557 of these patients were female, with the remaining two being male (Table 1). The calculated $\mathrm{BM}$ rates for patients with $\mathrm{HR}+/ \mathrm{HER} 2-$ disease, HER2+ disease, and TNBC were 14.2\% (160/1,130), $25.1 \%$ (296/1,177), and 20.0\% (92/461), respectively, with 11 cases of unknown molecular type. At the time of the most recent follow-up, $350(62.6 \%)$ patients had died.

\section{Patient treatment approaches}

At the time of BM diagnosis, 330 patients initially received 
Table 2 Efficacy of systemic drug therapy in 126 patients with new brain metastases

\begin{tabular}{|c|c|c|c|c|c|c|}
\hline Drug therapy & $\mathrm{CR}$ & PR & SD & $\mathrm{SD} \geq 6 \mathrm{~m}$ & PD & CBR \\
\hline Chemotherapy alone (14 cases, 14 schemes) & 1 & 3 & 9 & 4 & 1 & $50.0 \%$ \\
\hline $\begin{array}{l}\text { Chemotherapy + targeted therapy (macromolecule mAb + } \\
\text { TKI) ( } 4 \text { cases, } 4 \text { schemes) }\end{array}$ & 0 & 0 & 4 & 2 & 0 & $50.0 \%$ \\
\hline $\begin{array}{l}\text { Chemotherapy + targeted therapy (TKI) }(40 \text { cases, } 39 \\
\text { schemes) }\end{array}$ & 2 & 19 & 14 & 4 & 4 & $64.1 \%$ \\
\hline \multicolumn{7}{|l|}{ HER2-negative $(n=44)$} \\
\hline Endocrine therapy ( 11 cases, 12 schemes) & 1 & 1 & 8 & 5 & 2 & $58.3 \%$ \\
\hline
\end{tabular}

CR, complete response; PR, partial response; SD, stable disease; PD, progressive disease; CBR, clinical benefit rate; HER2, human epidermal growth factor receptor 2; TKI, tyrosine-kinase inhibitor; mAb, monoclonal antibody.

local brain treatment combined with systemic drug therapy (including 257 cases of whole-brain radiotherapy, 106 cases of conformal radiotherapy/gamma knife treatment, and 23 cases of local surgery). Of the remaining cases, 148 patients initially received systemic drug therapy (with 36 having also received local brain treatment by the time of the most recent follow-up), 64 patients declined further treatment (including 10 patients with an expected survival of $<3$ months), and 17 patients had an unknown treatment status.

Of the 148 patients that initially received systemic drug treatment (none of whom exhibited meningeal metastases), 40 had HR+/HER2 - disease, 93 had HER2+ disease, 14 had TNBC, and one had disease of an unknown molecular type. Following BM diagnosis, the median nonbrain local treatment time was 10.0 months (95\% CI: 7.811-12.189 months). The curative effect for 126 patients (139 schemes) with brain lesions was evaluated. The ORR for these patients was $25.2 \%(35 / 139)$, while the CBR was $43.9 \%(61 / 139)$, and the DCR was $80.6 \%(112 / 139)$. Of the 126 patients that received systemic drug therapy, 61 achieved clinical benefit, 39 of whom (63.9\%) were diagnosed with HER2+ disease (Table 2).

Treatment regimens for patients that achieved CR included aromatase inhibitor ( 1 case), capecitabine $(1$ case), capecitabine + pyrotinib ( 2 cases), and capecitabine + vinorelbine (1 case).

\section{Assessment of the effectiveness of different preferred treatment methods and factors affecting the BMOS of patients with $M B C-B M$}

The median follow-up duration for the present study was 28.0 months, while the median BMOS was 16.0 months. At the time of BM diagnosis, the systemic drug treatment group fared better than the local intracranial treatment group, and the BMOS of these two groups was 22.0 and 16.0 months, respectively. This difference was statistically significant $\left(\chi^{2}=7.743, \mathrm{P}=0.005\right)$ (Figure $\left.2 A\right)$.

The majority of patients who initially received systemic treatment had a diagnosis of HER2+ disease (62.8\%). For HER2 + MBC-BM patients, the BMOS in the systemic drug treatment group (93 cases) was significantly better than that in the local intracranial treatment group (168 cases) (34.0 vs. 18.0 months; $\chi^{2}=5.546, \mathrm{P}=0.019$ ) (Figure $2 B$ ).

Univariate analyses of factors revealed that DFS, Karnofsky performance status (KPS), molecular type, number of extracranial metastases (ECMs), BM location, number of BMs, neurological symptoms, and the preferred treatment approach were all associated with BMOS (Table 3).

A subsequent multivariate Cox regression analysis revealed that except molecular type, and number of BMs, DFS, KPS, number of extracranial metastases, BM location, neurological symptoms, and preferred treatment approach were all independently associated with patient BMOS (Table 4). 

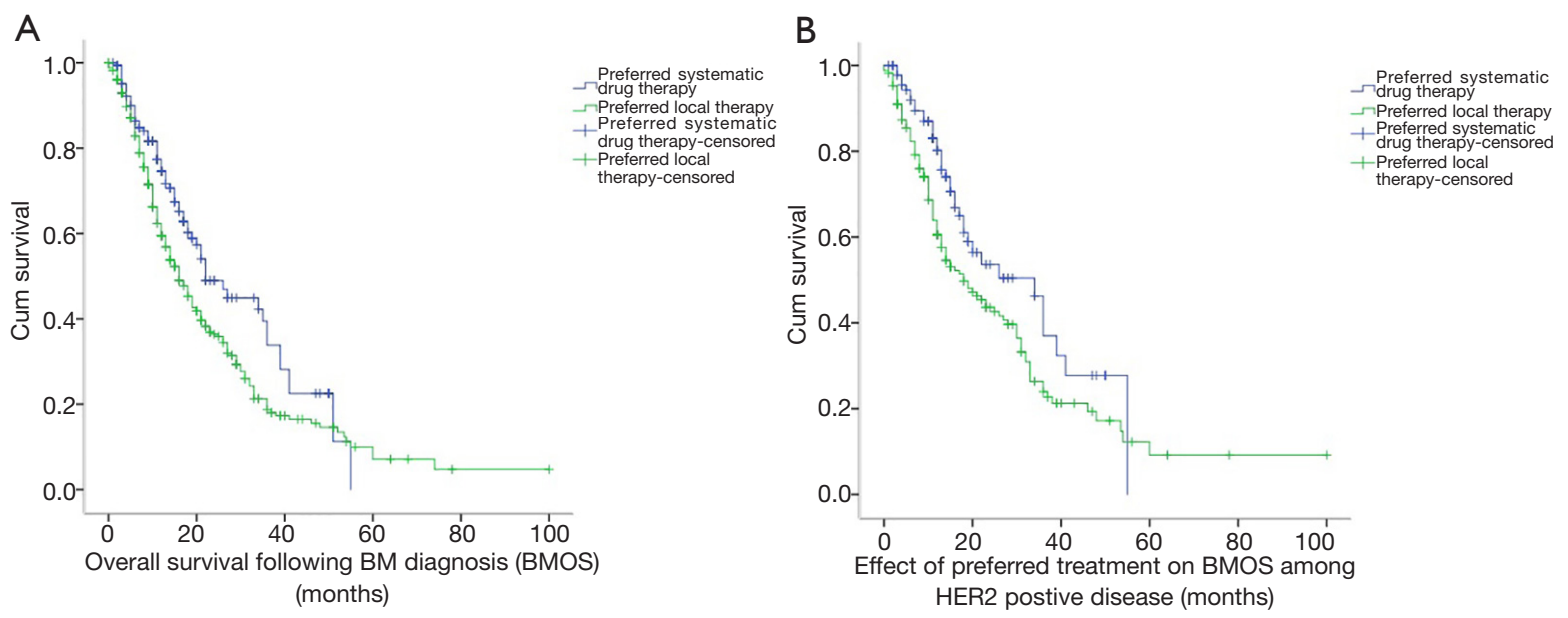

Figure 2 The Kaplan-Meier curve: (A) for the different preferred therapies from the initial diagnosis of brain metastasis (BM) to death due to any cause or most recent follow-up [overall survival following BM diagnosis (BMOS)]; (B) for the different preferred therapies on BMOS among HER2-positive patients.

A nomogram was constructed to calculate BMOS based upon the following eight factors: DFS, KPS, molecular type, ECMs, BM location, number of BMs, neurological symptoms, and the preferred treatment approach (Figure $3 A$ ). Based upon these logistic regression models, the prediction probability formula was established as follows: Logit $(\mathrm{BMOS})=3.756-0.5789 \times \mathrm{ECMs}-0.57308$ $x$ the location of $\mathrm{BM}+0.96 \times$ neurological symptoms -0.603 $x$ the preferred treatment $-0.094 \times$ number of BMs +0.376 $\times$ DFS $+0.456 \times$ molecular type $-2.2 \times$ KPS. The AUC for the entire patient cohort was 0.76 , indicating that the model was a good fit (Figure 3B).

To confirm the predictive utility of these eight variables, we conducted an additional internal model validation. ROC curves were generated to validate this nomogram in the validation set (AUC $=0.818,95 \%$ CI: $0.743-0.894$ ) (Figure $3 C$ ). As the AUC was $>0.8$, this indicated that the model exhibited good predictive power.

Neurological symptoms were diagnosed in a number of MBC-BM patients, including 120 cases of dizziness, 100 cases of headache, 61 cases of motor function or coordination/motor deficit, 22 cases of nausea and/or vomiting, 16 cases of epilepsy, 3 cases of diplopia, 3 cases of eye movement disorders, 3 cases of speech disorders, 3 cases of consciousness disturbance, and 2 cases of blurred vision or blindness.

At the time of the initial BM diagnosis, 341 patients were free of neurological symptoms, whereas 218 patients reported such symptoms. Patients without neurological symptoms diagnosed by regular screen had a significantly longer BMOS relative to patients with neurological symptoms (18.0 vs. 13.0 months; $\chi^{2}=11.371, \mathrm{P}=0.001$ ) (Figure $4 A$ ). There was no significant difference in the diagnosis of $\mathrm{BM}$ with or without neurological symptoms among patients with different molecular subtypes of breast cancer $\left(\chi^{2}=1.982, \mathrm{P}=0.371\right)$.

The median OS following recurrence or metastasis was 34.0 months. Patients without neurological symptoms exhibited a longer OS after metastasis than patients with neurological symptoms (36.0 vs. 29.0 months; $\chi^{2}=6.008$, $\mathrm{P}=0.014$ ) (Figure $4 B)$.

The median time without BM for these patients was 48.0 months (95\% CI: 42.461-53.539), and this duration was independently associated with disease subtypes as follows: 68.0 months for HR+/HER2 - type disease, 43.0 months for HER2+ type disease, and 36.0 months for TNBC $\left(\chi^{2}=26.051, \mathrm{P}<0.0001\right)$.

The median time from recurrence or metastasis to BM was 16.0 months (95\% CI: 13.242-18.758), and this duration was independently associated with disease subtypes as follows: 22.0 months for HR+/HER2 - type disease, 16.0 months for HER2+ type disease, and 12.0 months for $\operatorname{TNBC}\left(\chi^{2}=13.125, \mathrm{P}=0.001\right)$.

\section{Discussion}

As comprehensive treatment options for breast cancer patients continue to improve, patients will continue to live 
Table 3 Univariate analysis of factors associated with BMOS

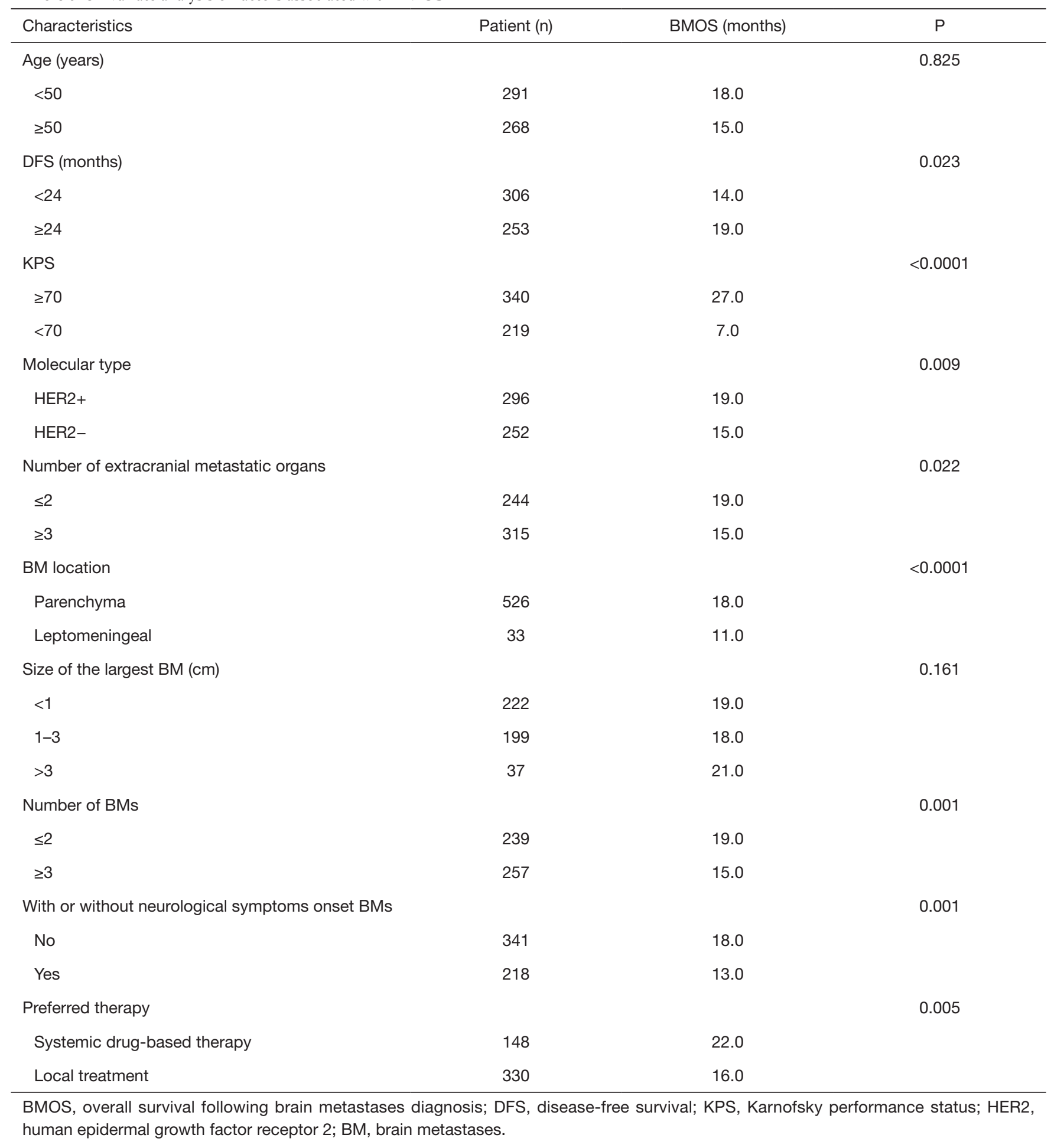


Table 4 Multivariate analysis of factors associated with BMOS

\begin{tabular}{lcc}
\hline Characteristics & $\mathrm{P}$ & $\mathrm{Hazard} \mathrm{ratio} \mathrm{(95 \%} \mathrm{Cl)}$ \\
\hline DFS & 0.05 & $1.272(1.000-1.616)$ \\
KPS & $<0.0001$ & $0.111(0.076-0.162)$ \\
Number of extracranial metastatic organs & 0.046 & $0.779(0.609-0.996)$ \\
BM location & 0.002 & $0.449(0.271-0.742)$ \\
With or without neurological symptoms onset BMs & $<0.0001$ & $4.079(2.772-6.001)$ \\
Preferred therapy-local treatment & $<0.0001$ & $0.348(0.228-0.532)$ \\
Preferred therapy-systemic drug therapy & $<0.0001$ & $0.455(0.314-0.658)$ \\
\hline
\end{tabular}

BMOS, overall survival following BM diagnosis; Cl, confidence interval; DFS, disease-free survival; KPS, Karnofsky performance status; $\mathrm{BM}$, brain metastases.

longer, which is likely to result in a higher BM diagnosed incidence. Prior reports have reported BM rates to be higher in patients with HR-, HER2+, and TNBC than in patients exhibiting other molecular disease subtypes $(5,24,25)$. Consistent with these prior reports, we observed the highest rate of BM in HER2+ MBC patients (25.1\%).

The median time between breast cancer diagnosis and $\mathrm{BM}$ has been reported as 41 months (26), although this report was based upon data generated two decades ago. In the present study, we instead found this median interval to be 48 months. The extension of time may be due to the development of novel drugs and the sustained improvements in breast cancer treatment in recent years.

Approximately $80 \%$ of BMs arise in the cerebral hemispheres, with the cerebellum being the second most common site and brainstem metastases being relatively uncommon. BMs are primarily multifocal metastases (78\%), with single metastases accounting for just $14 \%$ of total metastases (27). In the present study, we found that single BMs accounted for $29.7 \%$ of total metastases, with a fewer amount of BMs associated with a longer BMOS $\left(\chi^{2}=11.694\right.$, $\mathrm{P}=0.001)$, which is consistent with previous reports $(11,12)$.

Previous studies suggest that leptomeningeal metastasis is observed in approximately $5 \%$ of breast cancer cases (28). Kim et al. (29) reported that brain parenchymal metastases, meningeal metastases, and both brain parenchymal and meningeal metastases were detected in $79.5 \%$, $7.5 \%$, and $13 \%$ of patients, respectively. Patients with leptomeningeal metastases have a very poor prognosis with an estimated survival of just 2-4 months (30). Batista et al. (31) retrospectively conducted an analysis of MBC-BM patients over a 10 -year period and found that while leptomeningeal metastases were rare, they were associated with a poorer prognosis. Znidaric et al. (32) retrospectively assessed 423 breast cancer patients with brain parenchymal/meningeal metastases between 2005 and 2015. They found that the OS of patients was longer for those with a KPS $>70$, no extracranial lesions, or with only $\mathrm{BM}$ and no meningeal metastases. In the present study, only 33 cases of leptomeningeal metastasis were observed, accounting for $5.9 \%$ of overall cases. The BMOS of patients with leptomeningeal metastases was shorter than that of patients with brain parenchymal metastases, consistent with a poorer prognosis (11.0 vs. 18.0 months; $\left.\chi^{2}=15.452, \mathrm{P}<0.0001\right)$. Of these 33 patients, $42.4 \%$ reported no neurological symptoms, and their BMOS was longer than reported previously $(30,31)$, potentially because these metastases were detected early due to the regular cranial MRI screening. In the present cohort, we also found that all 17 cases of spinal cord metastasis occurred following brain parenchymal/ meningeal metastasis, with no patients exhibiting spinal cord metastases in isolation. As such, for patients with brain parenchymal/meningeal metastases, enhanced MRI scans of the spinal cord should be conducted as soon as possible if symptoms including bilateral lower limb weakness, paraplegia, or abnormal defecation and urination arise, to exclude the possibility of spinal cord metastasis.

Prior analyses have indicated that systemic drug therapy can have a beneficial effect on intracranial lesions (33-36). In the 126 patients in our study population that did not urgently require local intracranial treatment and where the effects of systemic drug treatment on intracranial lesions could be assessed, the CBR was $43.9 \%$, and the DCR was $80.6 \%$. We found that the median time from BM diagnosis to the need for local treatment due to worsening 


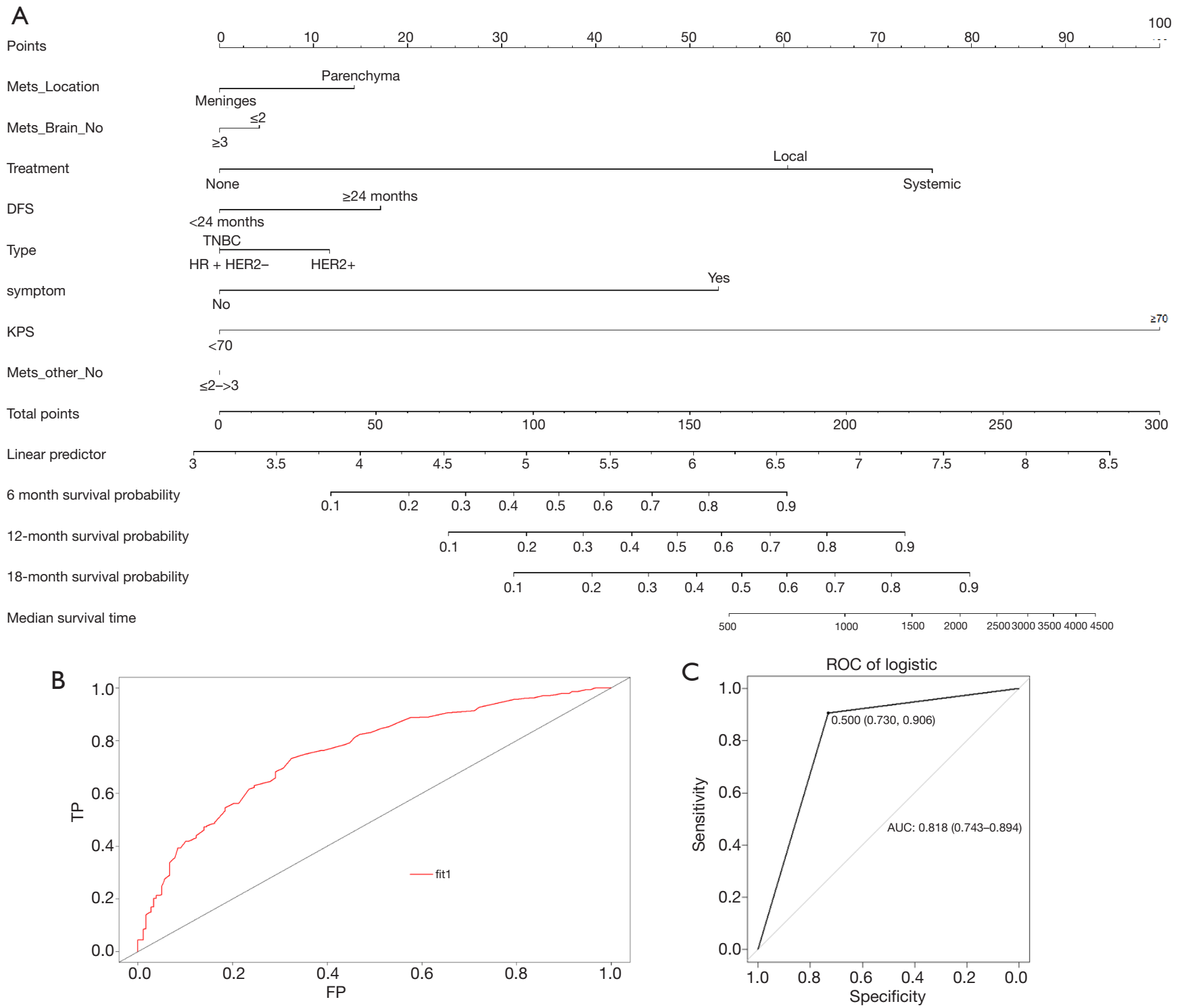

Figure 3 Nomogram to predict death from brain metastases (BM) in patients with BM from breast cancer (BC). (A) Nomogram for predicting the probability of overall survival following BM diagnosis (BMOS) using disease-free survival (DFS), Karnofsky performance score (KPS), molecular type, number of extracranial metastases, brain metastasis (BM) location, number of BMs, neurological symptoms, and the preferred treatment approach. (B) The area under the curve (AUC) in the nomogram is 0.76. (C) The receiver operating characteristic curve (ROC) of the validation sets in the nomogram is 0.818 .

disease symptoms was 10.0 months. At the time of initial BM diagnosis, patients who initially received systemic drug therapy exhibited a significantly longer BMOS than patients who received local treatment $\left(\chi^{2}=7.743, \mathrm{P}=0.005\right)$. The result was the same in the HER2+ subgroup. The BMOS of the 64 patients who declined further treatment following diagnosis was just 6 months. If the $\mathrm{BM}$ in these patients had been diagnosed earlier and treatment had been promptly initiated, their BMOS may have improved. Real-world clinical data indicate that systemic treatment is preferred treatment for patients with BM who have no neurological symptoms when brain metastases are detected early. Such treatment strategy does not shorten the patient's BMOS, conversely it can delay the time to local treatment. These data offer support for those patients whose disease progresses after the maximum dose of brain radiotherapy 

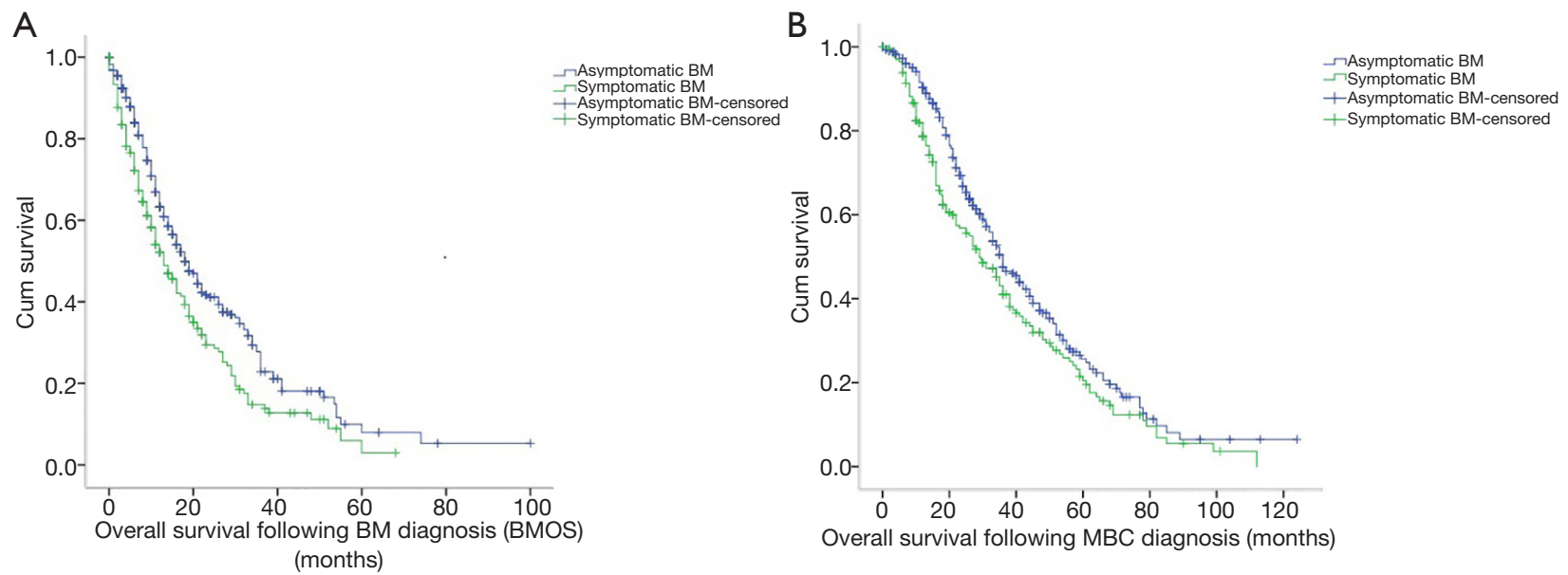

Figure 4 The Kaplan-Meier curve: (A) from the initial diagnosis of brain metastasis (BM) to death due to any cause or most recent followup [overall survival following BM diagnosis (BMOS)]; (B) from the initial diagnosis of metastatic breast cancer (MBC) to death due to any cause or most recent follow-up (OS).

has been given and who require subsequent systemic therapy.

Prior reports have indicated that BMOS typically ranges from 3.7-15 months, with TNBC having the worst prognosis with an average survival of just 4 months (37-39). Darlix et al. (39) reported that the median BMOS was 7.9 months and that this rate was independently associated with disease subtype; the median BMOS for patients with HER2+/HR+, HER2+/HR-, TNBC, and HER2-/HR+ disease was 18.9, 13.1, 4.4, and 7.1 months, respectively $(\mathrm{P}<0.0001)$. In the present study, the median BMOS was 16 months, and we also found it to be independently associated with disease subtype, TNBC had the worst prognosis, while the prognosis of HER2+ patients (particularly HR+/HER2 + patients) was longer (22.0 months). Our findings are consistent with the results published by Matsuo et al. (7), who found that HR-/HER2+ and $\mathrm{HR}+/ \mathrm{HER} 2+$ patients survived significantly longer than HER2 - patients (hazard ratio $=0.47, \mathrm{P}<0.001$ ). This may be attributable to the continued clinical application of increasingly efficacious anti-HER2 treatment regimens in recent years $(33-36,40)$.

Laakmann et al. (41) found that patients with asymptomatic BM detected through screening exhibited better survival outcomes than patients with symptomatic $\mathrm{BM}$, with respective median BMOS durations of 10.4 vs. 6.9 months. Consistent with this, we observed a significantly longer BMOS for patients with asymptomatic BM diagnosed by regular screen relative to patients with symptomatic BM (18.0 vs. 13.0 months; $\chi^{2}=11.371$, $\mathrm{P}=0.001)$. The rate of asymptomatic $\mathrm{BM}$ in this study $(61.1 \%)$ was higher than that reported previously (42), most likely because we conducted routine screening of patients via cranial MRI to detect $\mathrm{BM}$ at an early stage. In addition to this high rate of asymptomatic BM, we found that almost $40 \%$ of patients had $\leq 3 \mathrm{BMs}$ in number, and nearly $75 \%$ of patients exhibited BMs $<3 \mathrm{~cm}$ in size, with the corresponding BMOS for these patients being significantly longer than reported previously $(5,37,43)$. This suggests that regular cranial MRI screening can detect asymptomatic $\mathrm{BM}$ at an early stage, with the BMOS of those patients with asymptomatic BM being longer than that of individuals with symptomatic BM.

Many studies have reported that an asymptomatic status, good KPS at the time of BM onset, stable or better extracranial lesions, $\leq 4 \mathrm{BMs}$ in number, disease subtype, and HER2-overexpression are associated with a better prognosis $(7,44,45)$. Univariate analyses performed in the present study identified DFS ( $\geq 24$ months), KPS ( $\geq 70$ ), disease subtype (HER2+), ECMs $(\leq 2)$, BM location (brain parenchymal metastases), number of BMs $(\leq 2)$, asymptomatic status, and preferred systemic drug therapy as good prognostic factors. However, the number of BMs and the disease subtype were not significantly associated with BMOS in a subsequent multivariate analysis.

Though previous studies have constructed well-designed nomograms predicting BMOS $(19,20,46,47)$, the advantage of our nomogram is that it includes current treatment 
and medical examination strategies. Therefore, it may provide a more accurate assessment of BMOS probability according to the risk factors. Based upon our results, DFS, KPS, number of extracranial metastases, BM location, neurological symptoms, and the preferred treatment approach were all significant predictors of BMOS. Our nomogram may thus offer more reliable information when evaluating the prognosis of MBC-BM patients. Despite the advantages of the present study, this is a retrospective analysis with inherent selection bias. A variety of treatment modalities were applied during the lengthy period of the study [2010-2019], which implies that many changes in the treatment of $\mathrm{MBC}-\mathrm{BM}$ patients occurred during this time in terms of local treatment options and imaging methods to monitor disease progress. Thus, further prospective studies are warranted.

For the present study, BM diagnoses were based upon the guidance of doctors that were well-versed in radiological imaging, thus ensuring accuracy. However, this study is limited by the fact that it spans a 10 -year period. The molecular typing of some patients prior to 2011 was unknown, and some imaging films could no longer be accurately read, hampering efforts to diagnose BM accurately. However, the number of affected cases was small and unlikely to have affected our overall results. In addition, as many drugs are unable to cross the blood-brain barrier, the responses of intracranial and extracranial lesions to systemic drug therapy were frequently inconsistent. When extracranial lesions progress, systemic treatment plans need to be changed, potentially hampering efforts to observe the efficacy of such systemic treatment on intracranial lesions.

\section{Conclusions}

Systemic drug therapy has a beneficial effect on brain lesions, and effective treatment can delay local intracranial treatment. Cranial MRI screening can detect asymptomatic BM in MBC patients, particularly for those with HER2positive or TNBC subtypes, offering them an opportunity to receive systemic drug therapy and thereby prolong their survival. The use of regular examination cranial MRI scans to screen for asymptomatic BMs in breast cancer patients, and the clinical benefits of preferred systemic drug therapy for patients without an urgent need to undergo local brain therapy (especially in those subtypes where BM rates are high and more systemic drug treatment options are available), require multi-center, prospective studies for further verification. In the future, it will also be important to study the mechanisms of BM and to develop drugs that can more effectively treat intracranial lesions so as to improve the prognosis of patients with BM, particularly for those with HER2 - disease.

\section{Acknowledgments}

The authors would like to thank all the reviewers who participated in the review and MJEditor (www.mjeditor. com) for their linguistic assistance during the preparation of this manuscript.

Funding: None.

\section{Footnote}

Reporting Checklist: The authors have completed the STROBE reporting checklist. Available at https://dx.doi. org/10.21037/atm-21-3734

Data Sharing Statement: Available at https://dx.doi. org/10.21037/atm-21-3734

Conflicts of Interest: All authors have completed the ICMJE uniform disclosure form (available at https://dx.doi. org/10.21037/atm-21-3734). The authors have no conflicts of interest to declare.

Ethical Statement: The authors are accountable for all aspects of the work in ensuring that questions related to the accuracy or integrity of any part of the work are appropriately investigated and resolved. All procedures performed in this study involving human participants were in accordance with the Declaration of Helsinki (as revised in 2013). This study was approved by the medical ethics committee of The Affiliated Cancer Hospital of Zhengzhou University \& Henan Cancer Hospital (No. 2018160). Informed consent from patients was waived due to the retrospective nature of this study.

Open Access Statement: This is an Open Access article distributed in accordance with the Creative Commons Attribution-NonCommercial-NoDerivs 4.0 International License (CC BY-NC-ND 4.0), which permits the noncommercial replication and distribution of the article with the strict proviso that no changes or edits are made and the original work is properly cited (including links to both the formal publication through the relevant DOI and the license). 
See: https://creativecommons.org/licenses/by-nc-nd/4.0/.

\section{References}

1. Lin NU, Bellon JR, Winer EP. CNS metastases in breast cancer. J Clin Oncol 2004;22:3608-17.

2. Tabouret $\mathrm{E}$, Chinot $\mathrm{O}$, Metellus $\mathrm{P}$, et al. Recent trends in epidemiology of brain metastases: an overview. Anticancer Res 2012;32:4655-62.

3. Jeon W, Jang BS, Jeon SH, et al. Analysis of survival outcomes based on molecular subtypes in breast cancer brain metastases: A single institutional cohort. Breast J 2018;24:920-6.

4. Arvold ND, Oh KS, Niemierko A, et al. Brain metastases after breast-conserving therapy and systemic therapy: incidence and characteristics by biologic subtype. Breast Cancer Res Treat 2012;136:153-60.

5. Kennecke H, Yerushalmi R, Woods R, et al. Metastatic behavior of breast cancer subtypes. J Clin Oncol 2010;28:3271-7.

6. Cruz-Muñoz W, Kerbel RS. Preclinical approaches to study the biology and treatment of brain metastases. Semin Cancer Biol 2011;21:123-30.

7. Matsuo S, Watanabe J, Mitsuya K, et al. Brain metastasis in patients with metastatic breast cancer in the real world: a single-institution, retrospective review of 12-year followup. Breast Cancer Res Treat 2017;162:169-79.

8. Altundag K, Bondy ML, Mirza NQ, et al. Clinicopathologic characteristics and prognostic factors in 420 metastatic breast cancer patients with central nervous system metastasis. Cancer 2007;110:2640-7.

9. Miller KD, Weathers T, Haney LG, et al. Occult central nervous system involvement in patients with metastatic breast cancer: prevalence, predictive factors and impact on overall survival. Ann Oncol 2003;14:1072-7.

10. Ramakrishna N, Temin S, Chandarlapaty S, et al. Recommendations on disease management for patients with advanced human epidermal growth factor receptor 2-positive breast cancer and brain metastases: American Society of Clinical Oncology clinical practice guideline. J Clin Oncol 2014;32:2100-8.

11. Dawood S, Broglio K, Esteva FJ, et al. Survival among women with triple receptor-negative breast cancer and brain metastases. Ann Oncol 2009;20:621-7.

12. Gao C, Wang F, Suki D, et al. Effects of systemic therapy and local therapy on outcomes of 873 breast cancer patients with metastatic breast cancer to brain: MD Anderson Cancer Center experience. Int J Cancer
2021;148:961-70.

13. Lin $M$, Jin $Y$, Jin J, et al. A risk stratification model for predicting brain metastasis and brain screening benefit in patients with metastatic triple-negative breast cancer. Cancer Med 2020;9:8540-51.

14. Clayton AJ, Danson S, Jolly S, et al. Incidence of cerebral metastases in patients treated with trastuzumab for metastatic breast cancer. Br J Cancer 2004;91:639-43.

15. Steeg PS, Camphausen KA, Smith QR. Brain metastases as preventive and therapeutic targets. Nat Rev Cancer 2011;11:352-63.

16. Cardoso F, Senkus E, Costa A, et al. 4th ESO-ESMO International Consensus Guidelines for Advanced Breast Cancer (ABC 4)†. Ann Oncol 2018;29:1634-57.

17. Nabors LB, Portnow J, Ahluwalia M, et al. Central Nervous System Cancers, Version 3.2020, NCCN Clinical Practice Guidelines in Oncology. J Natl Compr Canc Netw 2020;18:1537-70.

18. Jiang Z, Song E, Wang X, et al. Guidelines of Chinese Society of Clinical Oncology (CSCO) on Diagnosis and Treatment of Breast Cancer (2020 version). Transl Breast Cancer Res 2020;1:27.

19. Sperduto PW, Kased N, Roberge D, et al. Effect of tumor subtype on survival and the graded prognostic assessment for patients with breast cancer and brain metastases. Int J Radiat Oncol Biol Phys 2012;82:2111-7.

20. Sperduto PW, Kased N, Roberge D, et al. Summary report on the graded prognostic assessment: an accurate and facile diagnosis-specific tool to estimate survival for patients with brain metastases. J Clin Oncol 2012;30:419-25.

21. Subbiah IM, Lei X, Weinberg JS, et al. Validation and development of a modifified breast graded prognostic assessment as a tool for survival in patients with breast cancer and brain metastases. J Clin Oncol 2015;33:2239-45.

22. Sperduto PW, Mesko S, Li J, et al. Beyond an Updated Graded Prognostic Assessment (Breast GPA): A Prognostic Index and Trends in Treatment and Survival in Breast Cancer Brain Metastases From 1985 to Today. Int J Radiat Oncol Biol Phys 2020;107:334-43.

23. Sperduto PW, Kased N, Roberge D, et al. The effect of tumor subtype on the time from primary diagnosis to development of brain metastases and survival in patients with breast cancer. J Neurooncol 2013;112:467-72.

24. Martin AM, Cagney DN, Catalano PJ, et al. Brain Metastases in Newly Diagnosed Breast Cancer: A Population-Based Study. JAMA Oncol 2017;3:1069-77.

25. Yan M, Lü HM, Liu ZZ, et al. High risk factors of brain 
metastases in 295 patients with advanced breast cancer. Chin Med J (Engl) 2013;126:1269-75.

26. Sen M, Demiral AS, Cetingöz R, et al. Prognostic factors in lung cancer with brain metastasis. Radiother Oncol 1998;46:33-8.

27. Wilhelm I, Molnár J, Fazakas C, et al. Role of the bloodbrain barrier in the formation of brain metastases. Int J Mol Sci 2013;14:1383-411.

28. Franzoi MA, Hortobagyi GN. Leptomeningeal carcinomatosis in patients with breast cancer. Crit Rev Oncol Hematol 2019;135:85-94.

29. Kim HJ, Im SA, Keam B, et al. Clinical outcome of central nervous system metastases from breast cancer: differences in survival depending on systemic treatment. J Neurooncol 2012;106:303-13.

30. Beauchesne P. Intrathecal chemotherapy for treatment of leptomeningeal dissemination of metastatic tumours. Lancet Oncol 2010;11:871-9.

31. Batista MV, Tomás T, Eiriz IF, et al. Brain metastasis of breast cancer: A 10-year single institution retrospective analysis. Ann Oncol 2019;30:iii56.

32. Znidaric T, Gugic J, Marinko T, et al. Breast cancer patients with brain metastases or leptomeningeal disease: 10 -year results of a national cohort with validation of prognostic indexes. Breast J 2019;25:1117-25.

33. Bachelot T, Romieu G, Campone M, et al. Lapatinib plus capecitabine in patients with previously untreated brain metastases from HER2-positive metastatic breast cancer (LANDSCAPE): a single-group phase 2 study. Lancet Oncol 2013;14:64-71.

34. Awada A, Colomer R, Bondarenko I, et al. Efficacy and CNS progression analysis from the randomized phase 2 trial of neratinib + paclitaxel vs trastuzumab + paclitaxel as first-line treatment for HER2 + metastatic breast cancer (NEfERTT). Ann N Y Acad Sci 2015;589:599-615.

35. Murthy RK, Loi S, Okines A, et al. Tucatinib, Trastuzumab, and Capecitabine for HER2-Positive Metastatic Breast Cancer. N Engl J Med 2020;382:597-609.

36. Yan M, Ouyang Q, Sun T, et al. Pyrotinib and capecitabine for HER2-positive metastatic breast cancer patients with previously untreated brain metastases: A single-group multicenter phase II study. ESMO2020 (Absract 1380, poster presentation).

37. Lin NU, Claus E, Sohl J, et al. Sites of distant recurrence and clinical outcomes in patients with metastatic triplenegative breast cancer: high incidence of central nervous system metastases. Cancer 2008;113:2638-45.

38. Niikura N, Hayashi N, Masuda N, et al. Treatment outcomes and prognostic factors for patients with brain metastases from breast cancer of each subtype: a multicenter retrospective analysis. Breast Cancer Res Treat 2014;147:103-12.

39. Darlix A, Louvel G, Fraisse J, et al. Impact of breast cancer molecular subtypes on the incidence, kinetics and prognosis of central nervous system metastases in a large multicentre real-life cohort. Br J Cancer 2019;121:991-1000.

40. Yan M, Bian L, Hu X, et al. Pyrotinib plus capecitabine for human epidermal factor receptor 2-positive metastatic breast cancer after trastuzumab and taxanes (PHENIX): a randomized, double-blind, placebo-controlled phase 3 study. Transl Breast Cancer Res 2020;1:13.

41. Laakmann E, Witzel I, Neunhöffer T, et al. Characteristics and Clinical Outcome of Breast Cancer Patients with Asymptomatic Brain Metastases. Cancers (Basel) 2020;12:2787.

42. Maurer C, Tulpin L, Moreau M, et al. Risk factors for the development of brain metastases in patients with HER2positive breast cancer. ESMO Open 2018;3:e000440.

43. Witzel I, Laakmann E, Weide R, et al. Treatment and outcomes of patients in the Brain Metastases in Breast Cancer Network Registry. Eur J Cancer 2018;102:1-9.

44. Braccini AL, Azria D, Thezenas S, et al. Prognostic factors of brain metastases from breast cancer: impact of targeted therapies. Breast 2013;22:993-8.

45. Leyland-Jones B. Human epidermal growth factor receptor 2-positive breast cancer and central nervous system metastases. J Clin Oncol 2009;27:5278-86.

46. Sperduto PW, Mesko S, Li J, et al. Survival in Patients With Brain Metastases: Summary Report on the Updated Diagnosis-Specific Graded Prognostic Assessment and Definition of the Eligibility Quotient. J Clin Oncol 2020;38:3773-84.

47. Ahn HK, Lee S, Park YH, et al. Prediction of outcomes for patients with brain parenchymal metastases from breast cancer (BC): a new BC-specific prognostic model and a nomogram. Neuro Oncol 2012;14:1105-13.

(English Language Editor: D. Fitzgerald)

Cite this article as: Niu L, Lv H, Zhang M, Zeng H, Wang L, Cui S, Liu Z, Yan M. Clinical diagnosis and treatment of breast cancer with brain metastases and establishment of a prognostic model: a 10-year, single-center, real-world study of 559 cases. Ann Transl Med 2021;9(16):1331. doi: 10.21037/atm-21-3734 